Please ensure that your abstract fits into one column on one page and complies with the Instructions to Authors available from the Abstract Submission web page.

\title{
Reconstruct the paleoclimate and paleoenvironment of Gongga Mountain, China
}

\author{
Yingqin WU ${ }^{1,2}$, Yongli WANG ${ }^{2 *}$, Tianzhu LEI ${ }^{1}$, Yanhong LIU $^{1}$, \\ Zhiyu WANG ${ }^{3}$,Yan $\mathrm{LIU}^{1}$, Yanqing XIA ${ }^{1 *}$ \\ ${ }^{1}$ Key Laboratory of Petroleum Resources Research, Gansu \\ Province/ Key Laboratory of Petroleum Resources Research, \\ Northwest Institute of Eco-Environment and Resources, \\ Chinese Academy of Sciences, Lanzhou, 730000, China \\ (*correspondence: yqxia@,1zb.ac.cn,wyl16800@1zb.ac.cn) \\ ${ }^{2}$ Institute of Geology and Geophysics, Chinese Academy of \\ Science, 100029, Beijing, China \\ ${ }^{3}$ Graduate School of the Chinese Academy of Sciences, Beijing \\ 100049, China \\ (wuyingqin001@163.com)
}

Some indexes of long-chain $\boldsymbol{n}$-alkanes reflect the relative proportion of low organisms such as bacteria, algae, and high plants. These indexes include the $\boldsymbol{n}$-alkane average chain length $(A C L)$, the ratios of trees to grasses $\left(n-C_{29} / n-C_{31}\right)$, the ratios of $\sum n-C_{21} / \sum n-C_{21+}$ in $n$-alkanes and a carbon preference index (CPI). These indexes were used as indicators of the source and evolution of organic matter and allowed the reconstruction of the local vegetation history and environmental conditions. In addition, the total carbonate isotope $\delta^{13} \mathrm{C}$ values can be used to estimate the relative contributions of $\mathrm{C}_{3}$ and $\mathrm{C}_{4}$ plants, infer paleoclimate changes, and to examine past primary productivity. The study aimed to reconstruct the paleoclimate and paleoenvironment on Gongga Mountain by measuring the carbon isotope composition and lipid biomarkers in the sediments. Consequently, in this study, we evaluated the hydrocarbon potential of the sediments and determined how lipid biomarkers and carbon isotopes can be used to delineate the source of organic matter and related processes. Furthermore, the proxy indexes of biomarkers, TOC and the $\delta^{13} \mathrm{C}$ of organic matter from samples from Gongga Mountain were measured and investigated for paleoclimatic and paleoenvironmental reconstruction. The results show that the organic matter on Gongga Mountain was derived from three sources, bacteria, algae, and higher plants. In addition, $\mathrm{C}_{3}$ plants were the dominant input.

Supported by NSFC No. 41772147, 41272147, 41831176; the Instrument Equipment Function Development Technology Innovation Project of Chinese Academy of Sciences. No. E0280101. 